\title{
Theoretical Investigation on the Electronic and Optical Properties of Poly(fluorenevinylene) Derivatives as Light-Emitting Materials
}

\author{
Thanisorn Yakhanthip, ${ }^{1}$ Nawee Kungwan, ${ }^{1}$ Jitrayut Jitonnom, ${ }^{2}$ Piched Anuragudom, ${ }^{3}$ \\ Siriporn Jungsuttiwong, ${ }^{4}$ and Supa Hannongbua ${ }^{5}$ \\ ${ }^{1}$ Department of Chemistry and Center for Innovation in Chemistry, Faculty of Science, Chiang Mai University, \\ Chiang Mai 50200, Thailand \\ ${ }^{2}$ Department of Chemistry, School of Science, University of Phayao, Phayao 56000, Thailand \\ ${ }^{3}$ Department of Chemistry, Faculty of Liberal Arts \& Science, Kasetsart University Kamphaeng Saen Campus, \\ Khamphaeng Saen, Nakhon Pathom 73140, Thailand \\ ${ }^{4}$ Center for Organic Electronics and Polymers, Department of Chemistry, Faculty of Science, Ubon Ratchathani University, \\ Ubon Ratchathani 34190, Thailand \\ ${ }^{5}$ Department of Chemistry, Faculty of Science, Kasetsart University, Jatuchak, Bangkok 10900, Thailand
}

Correspondence should be addressed to Nawee Kungwan, nawee@chiangmai.ac.th

Received 5 April 2011; Accepted 10 June 2011

Academic Editor: F. Yakuphanoglu

Copyright () 2011 Thanisorn Yakhanthip et al. This is an open access article distributed under the Creative Commons Attribution License, which permits unrestricted use, distribution, and reproduction in any medium, provided the original work is properly cited.

Density functional theory (DFT) and time-dependent DFT (TDDFT) were employed to study ground-state properties, HOMOLUMO gaps $\left(\Delta_{\mathrm{H}-\mathrm{L}}\right)$, excitation energies $\left(E_{g}\right)$, ionization potentials (IPs), and electron affinities (EA) for PFV-alt-PDONV and PFV-alt-PDIH-PPV having different alternating groups. Excited-state properties were investigated using configuration interaction singles (CISs) while fluorescence energies were calculated using TDDFT. The results show that PFV-alt-PDONV exhibits blueshifted energies for both HOMO-LUMO gaps $\left(\Delta_{\mathrm{H}-\mathrm{L}}\right)$ and excitation energies $\left(E_{g}\right)$ compared with PFV-alt-PDIH-PPV. The predicted IP and EA clearly indicate that PFV-alt-PDIH-PPV has both easier hole creation and electron injection than that of PFV-alt-PDONV. The maximal absorption wavelengths of all polymers are strongly assigned to $\pi \rightarrow \pi^{*}$ transition. The predicted radiative lifetimes of PFV-alt-PDONV and PFV-alt-PDIH-PPV for B3LYP/6-31G(d) are 0.36 and $0.61 \mathrm{~ns}$, respectively, indicating that PFV-alt-PDIH-PPV should have a better performance for long-time emission than that of PFV-alt-PDONV.

\section{Introduction}

In the last decade, conjugated polymers have been found to be interesting materials for electronic and optoelectronic devices, such as flat panel display (FPD) [1], transistors [2], and solar cells [3]. The great result is commonly used such as the light-emitting diodes (LEDs) applications [4]. Polyfluorenes (PFs) which are important relative classes of conjugated polymer materials were first fabricated in 1991 [5] into PLED with high-chemical and photochemical stabilities. Especially, their emission wavelengths span over the whole visible spectrum and also have high fluorescent efficiency [6-8]. However, there are two major drawbacks that restrict their potential applicability. The first problem is the injection of holes which is much faster than transporting electrons affecting high energy consumption in PLED device [9]. The second aspect is an aggregation in condensed phase leading to low fluorescence quantum yields [10]. In 2002 [11], however, these problems have been excluded by inserting vinylene unit into the main chain of polyfluorenes and generated good properties of new class molecules, namely, as polyfluorenyl-2,7-vinylenes (PFVs).

In general, the optical band gap of conjugated polymers can be controlled via the modification of their chemical structures. In order to achieve the better properties, the chemical structure can be improved through the following methodologies: first $[9,12]$ by improving $\pi$-overlap of polymer backbone, this can be done by maintaining rings in a 
coplanar arrangement. Second $[10,13,14]$ is by introducing electron donor and acceptor moieties into either side chain or main chain of a conjugated molecule which can directly affect ionization potential (IP) and electron affinity (EA) of polymer. Third [15-17] is by copolymerization of different conjugated units into the polymer backbone, and, therefore, the optical band gap will be suitably tuned.

Recently, theoretical quantum calculations have been the famous tools because they can be used to rationalize the properties of known polymers and also predict those of unknown ones to guidance observed experimental synthesis. These methods include (i) density functional theory (DFT) [18], a method including electron correlation based on density of electron, has been found to give satisfactory results especially electronic ground-state geometries prediction. (ii) Time-dependent DFT (TDDFT) [19], a developed tool from DFT, has been used to compute optical properties, including excitation energies, oscillator strengths, and electronic compositions. (iii) Single configuration interaction (CIS) [20], the lowest level of methods for studying the excited-state properties, has been used to investigate the excited-state structures.

In this work, we study the electronic and optical properties of PFV-alt-PDONV and PFV-alt-PDIH-PPV by considering the effect of the alternative units between dialkoxyl naphthalenevinylene unit in PFV-alt-PDONV and dialkoxyl phenylenevinylene unit in PFV-alt-PDIH-PPV (as shown in Figure 1). The calculated results of these polymers will help us to get insight into the detailed information on structural and optical properties of new conjugated polymers. Furthermore, the calculated results can be used as the screening tools in selecting the new backbone and alternating group for the new chemical modification in experimental synthesis.

\section{Computational Methods}

The ground-state geometries of the studied molecules, $n=1-4$, were fully optimized by using DFT//B3LYP/6$31 \mathrm{G}(\mathrm{d})[21,22]$. All calculations were performed without any symmetry constraints in gas phase. The alkyl groups on five-membered ring of fluorene ring were replaced with hydrogen atom while the alkoxyl groups on naphthalene and phenylene rings were substituted by methoxyl groups to reduce the computing time. It has been reported that the alkyl groups do not significantly affect on the equilibrium structure and optical property of fluorene-based polymer $[23,24]$. The excited-state geometries were optimized by ab initio CIS/6-31G(d) [25]. The transition energy, oscillator strength, and electronic transition were calculated at both ground-state and excited-state optimized geometries using TDDFT/B3LYP, and the results were compared with the available experimental data. The electronic properties as well as ionization potentials (IPs) and electron affinities (EAs) were carried out only in vertical excitation calculation (v; at the geometry of neutral molecule) using B3LYP/6-31G(d) $[9,26]$. Due to the computation cost increases rapidly from monomer to oligomers, IP, EA, and CIS calculations
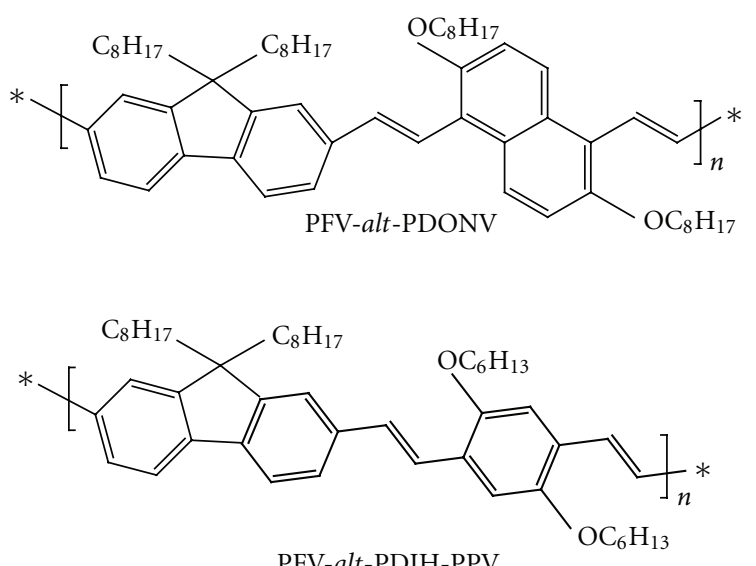

FIgURE 1: Conjugated polymers for theoretical analysis.

were studied in only three units of oligomers $(n=$ 1-3). Estimations of various properties of polymer such as $\Delta_{\mathrm{H}-\mathrm{L}}, E_{g}, \mathrm{IP}, \mathrm{EA}$, fluorescence energy, and radiative lifetime were obtained using the method based on oligomer approach by plotting the linearity between the calculated properties with the reciprocal of chain length and extrapolating to infinite chain length [27-29]. All quantum calculations were carried out using the Gaussian03 program package [30].

\section{Results and Discussions}

3.1. Ground-State Optimized Geometry. The selected optimized interring bond lengths and dihedral angles of these oligomers are listed in Table 1. As shown, it is observed that bond lengths and dihedral angles of each oligomer are not different when increasing oligomer size in both PFV-alt-PDONV and PFV-alt-PDIH-PPV implying that the structures of these polymers could be determined as their oligomers.

For PFV-alt-PDONV, the calculated dihedral angles between fluorene rings and vinylene units (F-V) and vinylene units and naphthalene rings $(\mathrm{V}-\mathrm{N})$ are average $11^{\circ}$ and $35^{\circ}$, respectively. It is found that the large dihedral angle of this polymer might come from the steric hindrance by rotation of methoxyl groups on naphthalene rings and the repulsion forces of two hydrogen atoms between naphthalene and vinylene rings.

For PFV-alt-PDIH-PPV, it is found that the dihedral angles between fluorene rings and vinylene units $(\mathrm{F}-\mathrm{V})$ and vinylene units and phenylene rings ( $\mathrm{V}-\mathrm{P})$ are average $5^{\circ}$ and $12^{\circ}$, respectively. There are steric effects from rotation of methoxyl groups on the phenylene rings and also the repulsion forces between hydrogen atom on phenylene ring and adjacent hydrogen atom on vinylene units as presented in PFV-alt-PDONV. However, these effects are less than that in PFV-alt-PDONV due to the distance between two adjacent hydrogen atoms on vinylene units and phenylene rings (V-P) are longer than that of between two adjacent hydrogen atoms on vinylene units and naphthalene rings $(\mathrm{V}-\mathrm{N})$. Comparing to PFV based [14], it is found that 
TABLE 1: Selected interring distances and dihedral angles of PFValt-PDONV and PFV-alt-PDIH-PPV $(n=1-4)$ obtained by B3LYP/6-31G(d,p).

\begin{tabular}{lcccccc}
\hline \multirow{2}{*}{ Oligomer } & \multicolumn{3}{c}{ Interring distances $(\AA)$} & \multicolumn{3}{c}{ Dihedral angles (deg) } \\
& F-V & V-N & V-P & F-V & V-N & V-P \\
\hline (PFV-alt- & & & & & & \\
PDONV) & & & & & & \\
$n=1$ & 1.4655 & 1.4693 & & 9.362 & 33.820 & \\
$n=2$ & 1.4656 & 1.4700 & & 12.021 & 35.066 & \\
$n=3$ & 1.4655 & 1.4699 & & 11.839 & 34.987 & \\
$n=4$ & 1.4656 & 1.4698 & & 11.986 & 34.786 & \\
\hline PFV-alt- & & & & & & \\
PDIH-PPV) & & & & & & 10.678 \\
$n=1$ & 1.4626 & & 1.4604 & 3.162 & & 14.374 \\
$n=2$ & 1.4621 & & 1.4603 & 5.603 & & 8.928 \\
$n=3$ & 1.4621 & & 1.4585 & 4.806 & & 12.652 \\
$n=4$ & 1.4622 & & 1.4588 & 6.487 & & \\
\hline
\end{tabular}

$\mathrm{F}$ is fluorene ring, $\mathrm{V}$ is vinylene unit, $\mathrm{N}$ is naphthalene ring, and $\mathrm{P}$ is phenylene ring.

the dihedral angles of these two polymers are larger than that of PFV based $\left(\sim 2^{\circ}\right)$ revealing that they should have more twisted structure. However, fortunately, the more noncoplanar structure of PFV-alt-PDONV and PFV-altPDIH-PPV might decrease the aggregation problems in their molecules when these polymers are applied in LED fabrications compared to PFV based. Moreover, the effect of different alternating group reveals that PFV-alt-PDONV has more noncoplanar geometry than PFV-alt-PDIH-PPV implying that PFV-alt-PDIH-PPV should have better $\pi$ delocalized electron affecting higher electron transporting performance.

3.2. Frontier Molecular Orbitals. The highest occupied molecular orbitals (HOMO) and the lowest unoccupied molecular orbitals (LUMO) are useful for understanding more details on excited-state properties. They can provide a reasonable qualitative indication of the subsequent excitation properties and the ability on electron or hole transport in feature of electron density contour. The contour plots of HOMO and LUMO orbitals of PFV-alt-PDONV and PFValt-PDIH-PPV $(n=4)$ by B3LYP/6-31G(d) are shown in Figure 2.

For PFV-alt-PDONV, the frontier molecular orbitals in oligomers do not spread over the whole $\pi$-conjugated backbone, but they predominantly localize on the naphthalene and less on fluorene rings in both HOMO and LUMO. These may be resulted from the large dihedral angle between vinylene units and naphthalene rings $(\mathrm{V}-\mathrm{N})$ and naphthalene rings and vinylene units $(\mathrm{N}-\mathrm{V})$ obstructing the electron transfer from HOMO to LUMO. The general HOMO shows antibonding between the bridges $\mathrm{C}-\mathrm{C}$ atoms of interring. Bonding is found between the bridge $\mathrm{C}=\mathrm{C}$ atoms and its conjoint atoms in naphthalene and phenylene rings. In contrary, there are interring bonding between the bridge $\mathrm{C}-\mathrm{C}$ atoms and antibonding between the bridge $\mathrm{C}=\mathrm{C}$ atoms in $\mathrm{LUMO}$.

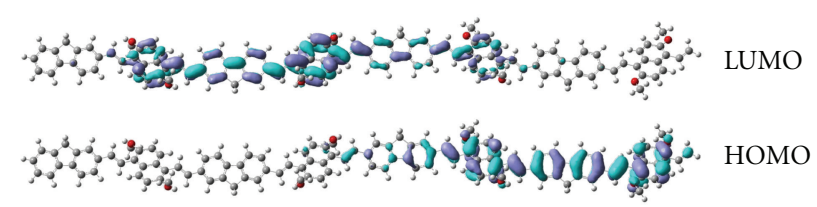

$(\mathrm{PFV} \text {-alt-PDONV })_{4}$

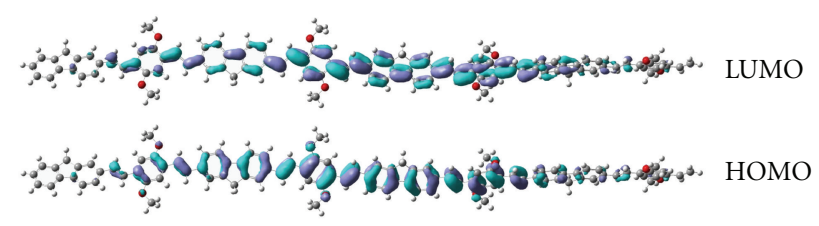

$(\mathrm{PFV} \text {-alt-PDIH-PPV })_{4}$

FIGURE 2: Frontier molecular orbitals of PFV-alt-PDONV and PFValt-PDIH-PPV $(n=4)$ by B3LYP/6-31G(d).

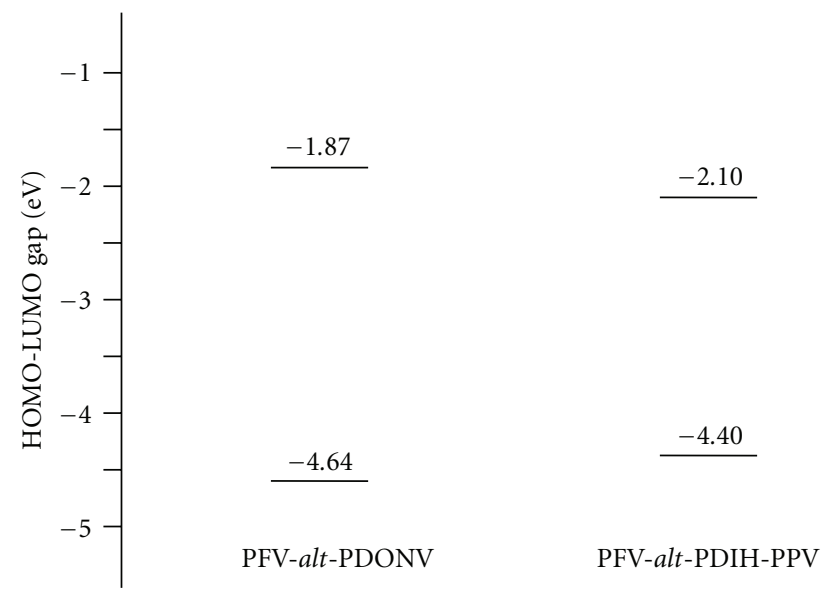

Figure 3: The HOMO and LUMO energies of PFV-alt-PDONV and PFV-alt-PDIH-PPV by B3LYP/6-31G(d).

For PFV-alt-PDIH-PPV, the delocalization of electron spreads over the whole $\pi$-conjugated backbone in both HOMO and LUMO. There is antibonding character between subunits and bonding character between $\mathrm{C}=\mathrm{C}$ atoms of intra-ring in $\mathrm{HOMO}$, but there is interring bonding in bridged $\mathrm{C}-\mathrm{C}$ atoms and antibonding between $\mathrm{C}=\mathrm{C}$ atoms in LUMO.

To gain insight into the effect of the alternating groups, the HOMO and LUMO energies of PFV-alt-PDONV and PFV-alt-PDIH-PPV were examined, and the results are depicted in Figure 3. From Figure 3, the HOMO energies of PFV-alt-PDONV and PFV-alt-PDIH-PPV are calculated to be -4.64 and $-4.40 \mathrm{eV}$, respectively. While the calculated LUMO energies of PFV-alt-PDONV and PFV-alt-PDIHPPV are -1.87 and $-2.10 \mathrm{eV}$, respectively. It is shown that the HOMO energies of PFV-alt-PDONV are lower than that of PFV-alt-PDIH-PPV about $0.24 \mathrm{eV}$, whereas the LUMO energies are higher than that of PFV-alt-PDIH-PPV about $0.23 \mathrm{eV}$. These results show that tuning the polymer backbone by adding the alternative dialkoxyl phenylenevinylene unit will more significantly stabilize LUMO and destabilize HOMO than that of dialkoxyl naphthalenevinylene unit 
indicating that phenylene ring should be an electronwithdrawing moiety. And these results should directly affect the band gap of PFV-alt-PDIH-PPV to be lower compared to that of PFV-alt-PDONV.

3.3. HOMO-LUMO Gaps and the Vertical Excitation Energies. There are two theoretical approaches used in this study to obtain the energy gaps. First approach is a crudely estimated from the different energies between HOMO and LUMO $\left(\Delta_{\mathrm{H}-\mathrm{L}}\right)$. Due to its simplicity, this approach can be used to provide valuable information on estimating band gaps in oligomers, polymers, and large systems. Another one is the vertical excitation energies $\left(E_{g}\right)$ method which is based on TDDFT. The TDDFT is a computational cost effective level and can be used to study the time-dependent properties by calculating the first dipole-allowed excitation energy of oligomers.

The $\Delta_{\mathrm{H}-\mathrm{L}}$ and $E_{g}$ of three polymers are listed in Table 2. The relationships between the calculated properties, $\Delta_{\mathrm{H}-\mathrm{L}}$ and $E_{g}$, with their inverse chain lengths are plotted in Figure 4. As shown in Table 2, the calculated $\Delta_{\mathrm{H}-\mathrm{L}}$ and $E_{g}$ of PFV-alt-PDONV are 2.77, and $2.40 \mathrm{eV}$ and PFV-altPDIH-PPV are 2.30 and $1.92 \mathrm{eV}$, respectively. Obviously, the results of both methods reveal that PFV-alt-PDONV has higher $\Delta_{\mathrm{H}-\mathrm{L}}$ and $E_{g}$ than that of PFV-alt-PDIH-PPV. These should be resulted from more twisted structure of PFV-altPDONV af-fecting lower electron transporting from $\mathrm{HOMO}$ to LUMO transition compared to PFV-alt-PDIH-PPV. Comparing to PFV based [14], it is found that PFV based (band gap at $2.13 \mathrm{eV}$ ) has lower and higher excitation energies than PFV-alt-PDONV and PFV-alt-PDIH-PPV with discrepancies about 0.27 and $0.21 \mathrm{eV}$, respectively. These results reveal that adding the alternative dialkoxyl phenylenevinylene unit in PFV-alt-PDIH-PPV should improve the efficiency of LEDs because it increases electron excitation probability and capability by considering from the valence band to the conduction band of molecules, which are the main ideal for LEDs modifying as energy aspect.

Moreover, we found that the vertical excitation energies $\left(E_{g}\right)$ from $S_{0} \rightarrow S_{1}$ agree well with experimental data [31, 32] just about $0.16 \mathrm{eV}$ different for PFV-alt-PDONV and $0.08 \mathrm{eV}$ for PFV-alt-PDIH-PPV while the HOMO-LUMO gaps $\left(\Delta_{\mathrm{H}-\mathrm{L}}\right)$ slightly overestimate the experimental data about $0.20 \mathrm{eV}$ for both PFV-alt-PDONV and PFV-alt-PDIH-PPV. This can be clarified that the columbic interaction is not taken into account in HOMO-LUMO gap calculation and also is not included in the significant contribution from some two-electron integrals [33]. In addition, the underestimation of TDDFT might be from two reasons; (i) DFT (B3LYP) system usually gives a small gap of materials and generates small excited energies on a large conjugated polymer [3436], and (ii) it may be caused by the solid-state effects (polarization effects and intermolecular packing forces) which are neglected in this calculation.

3.4. Ionization Potentials and Electron Affinities. Ionization potentials (IPs) and electron affinities (EAs) were employed to estimate the energy barrier for the injection of hole

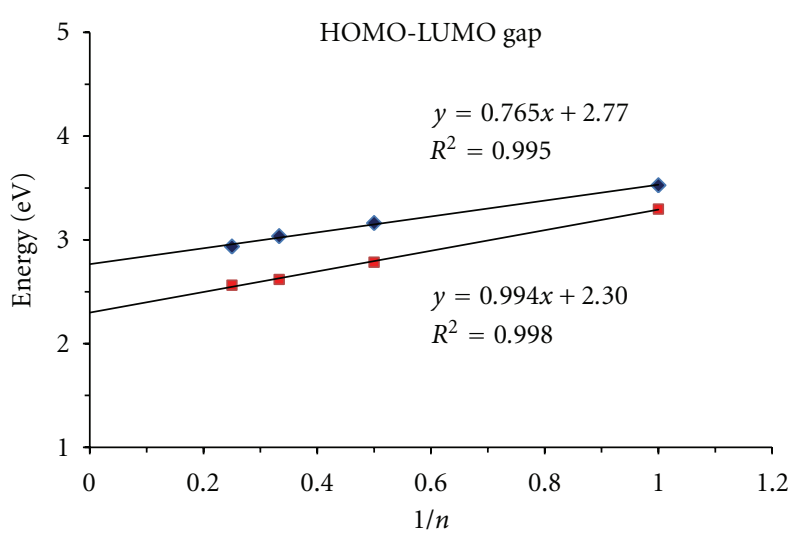

$(\mathrm{PFV}-\text { alt-PDONV })_{4}$

- PFV-alt-PDONV

- PFV-alt-PDIH-PPV

(a)

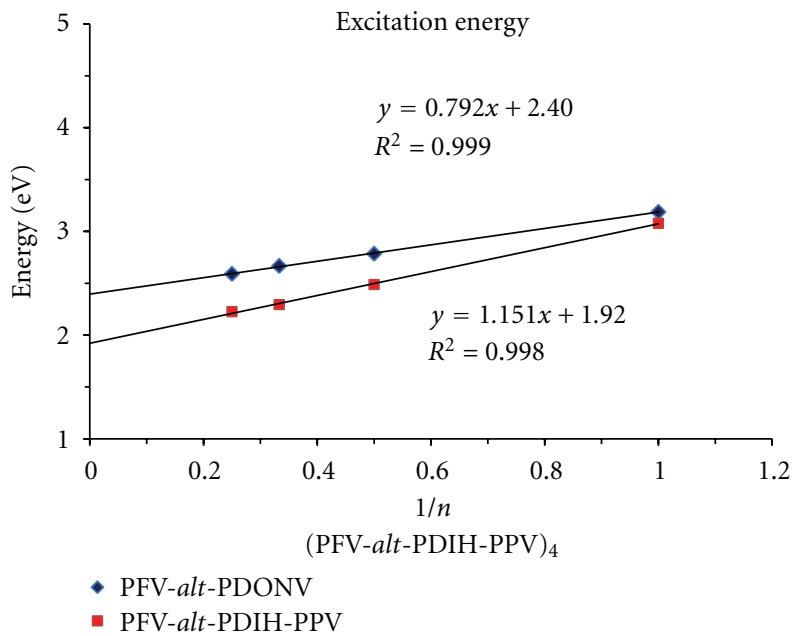

(b)

Figure 4: The HOMO-LUMO gaps $\left(\Delta_{H-L}\right)$ by B3LYP and the lowest excitation energies $\left(E_{g}\right)$ by TDDFT as a function of reciprocal chain length $n$ in oligomers of PFV-alt-PDONV and PFV-altPDIH-PPV.

and electron of PFV-alt-PDONV and PFV-alt-PDIH-PPV. The calculated results are shown in Table 3 . The energies required to create holes for PFV-alt-PDONV and PFV-altPDIH-PPV are calculated to be 5.12 and $4.98 \mathrm{eV}$, respectively. These values indicate that PFV-alt-PDIH-PPV has easier potential of hole injection and transportation compared to PFV-alt-PDONV. The extraction energy of an electron from the anion of PFV-alt-PDONV and PFV-alt-PDIH-PPV requires around 1.61 and $1.80 \mathrm{eV}$, respectively, indicating that the alternative dialkoxyl phenylenevinylene unit in PFV-altPDIH-PPV will improve the electron-accepting properties since their LUMO shows lower energies than that of PFV-altPDONV. These results imply that the injection of an electron from the cathode to the electron transporting layer of PFValt-PDIH-PPV is likely to be easier than that of PFV-altPDONV when these two polymers are fabricated into light emitting diode devices. 
TABLE 2: The HOMO-LUMO gaps $\left(\triangle_{H-L}\right)$ by B3LYP and the lowest excitation energies $\left(E_{g}\right)$ by TDDFT of PFV-alt-PDONV and PFValt-PDIH-PPV $(n=1-4)$.

\begin{tabular}{|c|c|c|c|}
\hline \multirow{2}{*}{ Oligomer } & \multirow[t]{2}{*}{$\Delta_{H-L}(\mathrm{eV})$} & \multicolumn{2}{|r|}{ TDDFT } \\
\hline & & $E_{g}(\mathrm{eV})$ & Wavelength (nm) \\
\hline \multicolumn{4}{|c|}{$(\mathrm{PFV}-\text { alt-PDONV })_{n}$} \\
\hline$n=1$ & 3.53 & 3.19 & 388.85 \\
\hline$n=2$ & 3.16 & 2.79 & 445.16 \\
\hline$n=3$ & 3.04 & 2.67 & 464.88 \\
\hline$n=4$ & 2.94 & 2.59 & 478.62 \\
\hline$n=\infty$ & 2.77 & 2.40 & 516.88 \\
\hline Exp. [31] & & 2.56 & 484.57 \\
\hline \multicolumn{4}{|c|}{$(\mathrm{PFV} \text {-alt-PDIH-PPV })_{n}$} \\
\hline$n=1$ & 3.30 & 3.08 & 402.78 \\
\hline$n=2$ & 2.79 & 2.49 & 498.24 \\
\hline$n=3$ & 2.62 & 2.29 & 540.35 \\
\hline$n=4$ & 2.56 & 2.23 & 556.89 \\
\hline$n=\infty$ & 2.30 & 1.92 & 646.09 \\
\hline Exp. [32] & & 2.10 & 592.00 \\
\hline
\end{tabular}

TABLE 3: Ionization potentials (IPs) and electron affinities (EAs) for PFV-alt-PDONV and PFV-alt-PDIH-PPV by B3LYP/6$31 \mathrm{G}(\mathrm{d})(\mathrm{eV})(n=1-3)$.

\begin{tabular}{lcc}
\hline Oligomer & $\mathrm{IP}(\mathrm{v})$ & $\mathrm{EA}(\mathrm{v})$ \\
\hline$(\mathrm{PFV}-\text { alt } \text {-PDONV })_{n}$ & & \\
$n=1$ & 6.28 & 0.47 \\
$n=2$ & 5.75 & 1.02 \\
$n=3$ & 5.48 & 1.24 \\
$n=\infty$ & 5.12 & 1.61 \\
\hline (PFV-alt -PDIH-PPV $)_{n}$ & & \\
$n=1$ & 6.13 & 0.46 \\
$n=2$ & 5.50 & 1.11 \\
$n=3$ & 5.40 & 1.37 \\
$n=\infty$ & 4.98 & 1.80 \\
\hline
\end{tabular}

3.5. Absorption Spectra. The TDDFT/B3LYP/6-31G(d) was employed to obtain the energy of the singlet-singlet electronic transitions as well as transition energies, oscillator strengths, and main configurations for five singlet-excited states of PFV-alt-PDONV and PFV-alt-PDIH-PPV, and the results are reported in Tables 4 and 5, respectively. As shown, TDDFT method shows the strongest excitation from $S_{0} \rightarrow S_{1}$ state and gives a good interpretive transition to the promotion of an electron from HOMO to LUMO. There are two interesting trends on oscillator strength $(f)$ in these tables; (i) the oscillator strengths $(f)$ in $S_{0} \rightarrow S_{1}$ state have the largest value in all series of all oligomers; (ii) the tendency of oscillator strengths $(f)$ increases with extending the conjugation length. Moreover, all electronic transitions of each oligomer are found to be $\pi-\pi^{*}$ transition character.

The maximal absorption wavelengths of PFV-altPDONV and PFV-alt-PDIH-PPV from TDDFT method are calculated to be 516.88 and $646.09 \mathrm{~nm}$, respectively. The absorption wavelength of PFV-alt-PDONV exhibits blue shifted corresponding to its more twisted structure compared to PFV-alt-PDIH-PPV. The absorption wavelength of $S_{0} \rightarrow$ $S_{1}$ transition is the longest among the five electronic transitions in all oligomers. Moreover, there is a trend showing that the absorption wavelength increases with extending molecular sizes as in the case of the oscillator strengths. Furthermore, we found that the oscillator strengths $(f)$ at $S_{0} \rightarrow S_{1}$ transition of each oligomer of PFV-alt-PDIH-PPV are bigger than that of PFV-alt-PDONV indicating that $\pi-\pi^{*}$ transition of PFV-alt-PDIH-PPV is stronger. These results confirm again that PFV-alt-PDIH-PPV should have better performance than PFV-alt-PDONV based on low energy consumption and high intensity absorption.

3.6. Excited-State Properties. The properties on the excited state were carried out by using configuration interaction singles (CIS). CIS is the cheapest method with reasonable accuracy for studying the excited-state properties. However, the prediction on CIS is not accuracy enough due to the neglecting of electron correlations [26]. Although CIS is not realizable level, but it can still be used as a qualitative tool to predict some tendency of excited-state properties [37]. The structures of all oligomers were optimized at CIS/6-31G(d). To reduce the expensive computing cost, all polymers were optimized only three units of oligomers $(n=1-3)$.

The prediction of different bond lengths between the ground $\left(S_{0}\right)$ and excited state $\left(S_{1}\right)$ can be studied by considering MO nodal patterns. The optimized excited structures $\left(S_{1}\right)$ by CIS/6-31G(d) were compared with the ground-state structure $\left(S_{0}\right)$ by HF/6-31G(d) in all oligomers. The comparisons of bond lengths between the excited and ground state of PFV-alt-PDONV and PFV-alt-PDIH-PPV are depicted in Figure 5. As shown in Figure 5, some bond lengths are lengthened but some bonds are shortened. The HOMO has node across $r(5,6), r(7,8), r(9,10), r(12,13)$, $r(11,14), r(15,16), r(19,20), r(24,25), r(21,28), r(29,30)$, $r(31,32), r(34,35), r(37,38), r(40,41), r(39,43), r(44,45)$, $r(48,49)$, and $r(53,54)$ bonds for PFV-alt-PDONV and $r(5,6), r(7,8), r(12,13), r(9,10), r(11,14), r(15,16), r(20,21)$, $r(17,18), r(19,24), r(25,26), r(27,28), r(30,31), r(29,32)$, $r(33,34), r(36,37), r(35,39), r(40,41), r(45,46), r(42,43)$, and $r(44,49)$ bonds for PFV-alt-PDIH-PPV while all oligomers have bonding in LUMO.

In opposite, the HOMO has bonding across $r(4,5)$, $r(6,7), r(2,7), r(8,13), r(8,9), r(10,11), r(11,12), r(14,15)$, $r(16,25), r(23,24), r(18,19), r(20,21), r(28,29), r(30,31)$, $r(30,35), r(32,33), r(33,34), r(36,37), r(36,41), r(38,39)$, $r(39,40), r(43,44), r(45,46), r(45,54), r(47,48), r(49,50)$, and $r(53,52)$ bonds for PFV-alt-PDONV and $r(4,5), r(6,7)$, $r(2,7), r(8,9), r(8,13), r(10,11), r(11,12), r(14,15), r(16,17)$, $r(16,21), r(18,19), r(19,20), r(24,25), r(26,27), r(26,31)$, $r(28,29), r(29,30), r(32,33), r(32,37), r(34,35), r(35,36)$, $r(39,40), r(41,46), r(41,42)$, and $r(44,45)$ for PFV-alt-PDIHPPV, but the LUMO has nodes across over these regions.

In addition, the fluorescence energies of all oligomers were estimated using TDDFT calculation on B3LYP/6$31 \mathrm{G}(\mathrm{d})$, and the results are summarized in Table 6. From Table 6, the fluorescence energies of each oligomer show similar tendency with the absorption wavelengths $\left(\lambda_{\mathrm{abs}}\right)$ in which 
TABLE 4: Electronic transition data obtained by TDDFT method for PFV-alt-PDONV $(n=1-4)$ at B3LYP/6-31G(d).

\begin{tabular}{|c|c|c|c|}
\hline Electronic transitions & Wavelengths (nm) & $f$ & Main configurations \\
\hline \multicolumn{4}{|l|}{ PFV-alt-PDONV } \\
\hline$S_{0} \rightarrow S_{1}$ & 388.85 & 1.0066 & $\mathrm{H} \rightarrow \mathrm{L}(85 \%)$ \\
\hline$S_{0} \rightarrow S_{2}$ & 339.28 & 0.0099 & $\mathrm{H}-1 \rightarrow \mathrm{L}(51 \%)$ \\
\hline$S_{0} \rightarrow S_{3}$ & 320.33 & 0.2709 & $\mathrm{H} \rightarrow \mathrm{L}+1(37 \%)$ \\
\hline$S_{0} \rightarrow S_{4}$ & 307.31 & 0.0336 & $\mathrm{H}-2 \rightarrow \mathrm{L}(52 \%)$ \\
\hline$S_{0} \rightarrow S_{5}$ & 294.86 & 0.0045 & $\mathrm{H} \rightarrow \mathrm{L}+2(68 \%)$ \\
\hline \multicolumn{4}{|l|}{$(\mathrm{PFV}-\text { alt }-\mathrm{PDONV})_{2}$} \\
\hline$S_{0} \rightarrow S_{1}$ & 445.16 & 2.4685 & $\mathrm{H} \rightarrow \mathrm{L}(87 \%)$ \\
\hline$S_{0} \rightarrow S_{2}$ & 398.95 & 0.0087 & $\mathrm{H} \rightarrow \mathrm{L}+1(77 \%)$ \\
\hline$S_{0} \rightarrow S_{3}$ & 397.57 & 0.0474 & $\mathrm{H}-1 \rightarrow \mathrm{L}(73 \%)$ \\
\hline$S_{0} \rightarrow S_{4}$ & 368.08 & 0.0258 & $\mathrm{H} \rightarrow \mathrm{L}+2(52 \%)$ \\
\hline$S_{0} \rightarrow S_{5}$ & 363.98 & 0.6254 & $\mathrm{H}-1 \rightarrow \mathrm{L}+1(86 \%)$ \\
\hline \multicolumn{4}{|l|}{$(\mathrm{PFV}-\text { alt-PDONV })_{3}$} \\
\hline$S_{0} \rightarrow S_{1}$ & 464.88 & 3.8927 & $\mathrm{H} \rightarrow \mathrm{L}(67 \%)$ \\
\hline$S_{0} \rightarrow S_{2}$ & 434.31 & 0.0639 & $\mathrm{H}-1 \rightarrow \mathrm{L}(52 \%)$ \\
\hline$S_{0} \rightarrow S_{3}$ & 421.81 & 0.2199 & $\mathrm{H} \rightarrow \mathrm{L}+1(50 \%)$ \\
\hline$S_{0} \rightarrow S_{4}$ & 409.94 & 0.4292 & $\mathrm{H}-1 \rightarrow \mathrm{L}+1(66 \%)$ \\
\hline$S_{0} \rightarrow S_{5}$ & 399.71 & 0.0004 & $\mathrm{H}-2 \rightarrow \mathrm{L}(50 \%)$ \\
\hline \multicolumn{4}{|l|}{$(\mathrm{PFV}-\text { alt-PDONV })_{4}$} \\
\hline$S_{0} \rightarrow S_{1}$ & 478.62 & 4.9660 & $\mathrm{H} \rightarrow \mathrm{L}(37 \%)$ \\
\hline$S_{0} \rightarrow S_{2}$ & 454.05 & 0.2804 & $\mathrm{H}-1 \rightarrow \mathrm{L}(32 \%)$ \\
\hline$S_{0} \rightarrow S_{3}$ & 436.32 & 0.5958 & $\mathrm{H} \rightarrow \mathrm{L}(43 \%)$ \\
\hline$S_{0} \rightarrow S_{4}$ & 432.48 & 0.2016 & $\mathrm{H}-1 \rightarrow \mathrm{L}+1(47 \%)$ \\
\hline$S_{0} \rightarrow S_{5}$ & 422.51 & 0.0040 & $\mathrm{H} \rightarrow \mathrm{L}+2(52 \%)$ \\
\hline
\end{tabular}

TABLE 5: Electronic transition data obtained by TDDFT method for PFV-alt-PDIH-PPV $(n=1-4)$ at B3LYP/6-31G(d).

\begin{tabular}{|c|c|c|c|}
\hline Electronic transitions & Wavelengths (nm) & $f$ & Main configurations \\
\hline \multicolumn{4}{|l|}{ PFV-alt-PDIH-PPV } \\
\hline$S_{0} \rightarrow S_{1}$ & 402.78 & 1.3722 & $\mathrm{H} \rightarrow \mathrm{L}(80 \%)$ \\
\hline$S_{0} \rightarrow S_{2}$ & 341.10 & 0.2476 & $\mathrm{H}-1 \rightarrow \mathrm{L}(85 \%)$ \\
\hline$S_{0} \rightarrow S_{3}$ & 313.14 & 0.0576 & $\mathrm{H} \rightarrow \mathrm{L}+1(62 \%)$ \\
\hline$S_{0} \rightarrow S_{4}$ & 301.95 & 0.0012 & $\mathrm{H} \rightarrow \mathrm{L}+2(72 \%)$ \\
\hline$S_{0} \rightarrow S_{5}$ & 282.01 & 0.0228 & $\mathrm{H}-2 \rightarrow \mathrm{L}(29 \%)$ \\
\hline \multicolumn{4}{|l|}{$(\mathrm{PFV}-\text { alt-PDIH-PPV })_{2}$} \\
\hline$S_{0} \rightarrow S_{1}$ & 498.24 & 3.3091 & $\mathrm{H} \rightarrow \mathrm{L}(86 \%)$ \\
\hline$S_{0} \rightarrow S_{2}$ & 430.94 & 0.0006 & $\mathrm{H}-1 \rightarrow \mathrm{L}(66 \%)$ \\
\hline$S_{0} \rightarrow S_{3}$ & 408.82 & 0.0044 & $\mathrm{H} \rightarrow \mathrm{L}+1(57 \%)$ \\
\hline$S_{0} \rightarrow S_{4}$ & 376.04 & 0.5267 & $\mathrm{H}-1 \rightarrow \mathrm{L}+1(84 \%)$ \\
\hline$S_{0} \rightarrow S_{5}$ & 369.20 & 0.0039 & $\mathrm{H}-2 \rightarrow \mathrm{L}(68 \%)$ \\
\hline \multicolumn{4}{|l|}{$(\mathrm{PFV} \text {-alt-PDIH-PPV })_{3}$} \\
\hline$S_{0} \rightarrow S_{1}$ & 540.35 & 4.9913 & $\mathrm{H} \rightarrow \mathrm{L}(86 \%)$ \\
\hline$S_{0} \rightarrow S_{2}$ & 470.79 & 0.0004 & $\mathrm{H}-1 \rightarrow \mathrm{L}(81 \%)$ \\
\hline$S_{0} \rightarrow S_{3}$ & 466.44 & 0.0077 & $\mathrm{H} \rightarrow \mathrm{L}+1(76 \%)$ \\
\hline$S_{0} \rightarrow S_{4}$ & 433.73 & 0.0671 & $\mathrm{H}-2 \rightarrow \mathrm{L}(70 \%)$ \\
\hline$S_{0} \rightarrow S_{5}$ & 431.29 & 0.9033 & $\mathrm{H}-1 \rightarrow \mathrm{L}+1(75 \%)$ \\
\hline \multicolumn{4}{|l|}{$(\mathrm{PFV}-\text { alt-PDIH-PPV })_{4}$} \\
\hline$S_{0} \rightarrow S_{1}$ & 556.89 & 6.7096 & $\mathrm{H} \rightarrow \mathrm{L}(81 \%)$ \\
\hline$S_{0} \rightarrow S_{2}$ & 502.80 & 0.0003 & $\mathrm{H}-1 \rightarrow \mathrm{L}(48 \%)$ \\
\hline$S_{0} \rightarrow S_{3}$ & 486.97 & 0.0003 & $\mathrm{H} \rightarrow \mathrm{L}+1(53 \%)$ \\
\hline$S_{0} \rightarrow S_{4}$ & 463.66 & 1.2450 & $\mathrm{H}-1 \rightarrow \mathrm{L}+1(80 \%)$ \\
\hline$S_{0} \rightarrow S_{5}$ & 459.86 & 0.0007 & $\mathrm{H}-2 \rightarrow \mathrm{L}(68 \%)$ \\
\hline
\end{tabular}



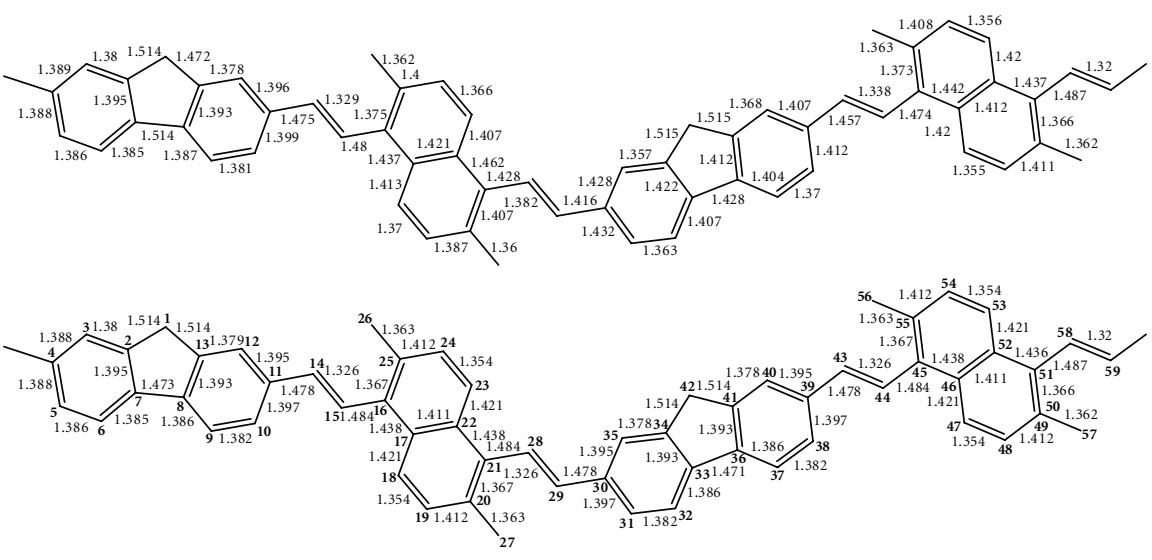

PFV-alt-PDONV
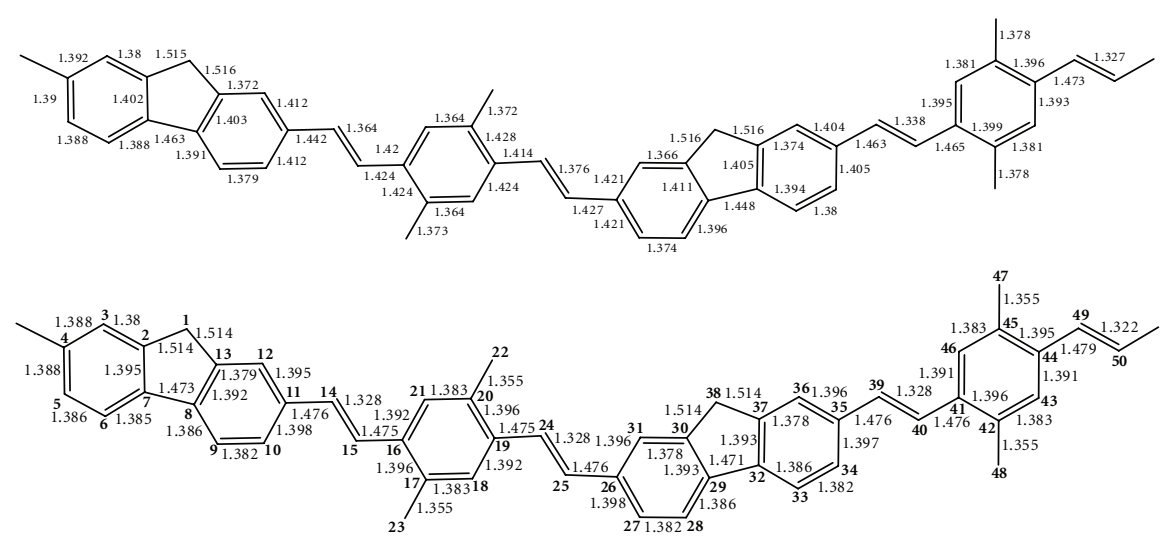

PFV-alt-PDIH-PPV

FIGURE 5: Comparison of the excited structures $\left(S_{1}\right)$ by CIS/6-31G(d) with the ground structure $\left(S_{0}\right)$ by HF/6-31G(d) $(n=2)$ for PFV-altPDONV and PFV-alt-PDIH-PPV.

TABLE 6: Fluorescence energies and radiative lifetime of PFV-alt-PDONV and PFV-alt-PDIH-PPV $(n=1-3)$ obtained by TDDFT//B3LYP/6$31 \mathrm{G}(\mathrm{d})$.

\begin{tabular}{|c|c|c|c|c|c|}
\hline Oligomer & Electronic transition & $f$ & Fluorescence energies $(\mathrm{eV})$ & Lifetime (ns) & Main configurations \\
\hline \multicolumn{6}{|c|}{$\overline{(\mathrm{PFV}-\text { alt-PDONV })_{n}}$} \\
\hline$n=1$ & $S_{1} \rightarrow S_{0}$ & 1.2498 & 2.71 & 2.51 & $\mathrm{H} \rightarrow \mathrm{L}(76 \%)$ \\
\hline$n=2$ & $S_{1} \rightarrow S_{0}$ & 2.5731 & 2.56 & 1.37 & $\mathrm{H} \rightarrow \mathrm{L}(82 \%)$ \\
\hline$n=3$ & $S_{1} \rightarrow S_{0}$ & 3.1934 & 2.54 & 1.12 & $\mathrm{H} \rightarrow \mathrm{L}(82 \%)$ \\
\hline$n=\infty$ & & & 2.45 & 0.36 & \\
\hline Exp. [31] & & & 2.37 & & \\
\hline \multicolumn{6}{|c|}{$(\mathrm{PFV}-\text { alt-PDIH-PPV })_{n}$} \\
\hline$n=1$ & $S_{1} \rightarrow S_{0}$ & 1.5925 & 2.75 & 1.91 & $\mathrm{H} \rightarrow \mathrm{L}(74 \%)$ \\
\hline$n=2$ & $S_{1} \rightarrow S_{0}$ & 3.4649 & 2.28 & 1.28 & $\mathrm{H} \rightarrow \mathrm{L}(79 \%)$ \\
\hline$n=3$ & $S_{1} \rightarrow S_{0}$ & 4.7141 & 2.17 & 1.04 & $\mathrm{H} \rightarrow \mathrm{L}(81 \%)$ \\
\hline$n=\infty$ & & & 1.86 & 0.61 & \\
\hline Exp. [32] & & & 1.85 & & \\
\hline
\end{tabular}

decrease with elongation of conjugation lengths. The fluorescence energies on the highest oscillator strength of each oligomer are assigned to $S_{1} \rightarrow S_{0}$ transition corresponding to $\mathrm{HOMO} \rightarrow$ LUMO configuration. The extrapolated fluorescence energies of PFV-alt-PDONV and PFV-alt-PDIH-PPV are 2.44 and $1.86 \mathrm{eV}$, respectively. Obviously, the predicted fluorescence energies by B3LYP/6-31G(d) excellently agree with the experimental data $[31,32]$ with discrepancies within $0.08 \mathrm{eV}$ for PFV-alt-PDONV and $0.01 \mathrm{eV}$ for PFV-alt-PDIHPPV. 
Finally, the fluorescence energies and oscillator strengths were used to calculate the radiative lifetime by using the Einstein transition probabilities in the following formula (in au.) [38]

$$
\tau=\frac{c^{3}}{2\left(E_{\mathrm{Flu}}\right)^{2} f},
$$

where $c$ is the velocity of light, $E_{\mathrm{Flu}}$ is the fluorescence transition energy, and $f$ is oscillator strength.

The predicted radiative lifetimes are collected in Table 6 . It is found that the radiative lifetime decreases with elongation of oligomer chain. The extrapolated lifetime of PFValt-PDONV and PFV-alt-PDIH-PPV by TDDFT//B3LYP/6$31 \mathrm{G}(\mathrm{d})$ are 0.36 and $0.61 \mathrm{eV}$, respectively. This result shows that the radiative lifetime of PFV-alt-PDONV is slightly shorter than that of PFV-alt-PDIH-PPV about $0.25 \mathrm{~ns}$ implying that PFV-alt-PDIH-PPV would have higher efficiency than PFV-alt-PDONV due to long-time emission of electrons. As to PFV based [14], the radiative lifetime is predicted to be $0.60 \mathrm{~ns}$ which is slightly longer than that of PFV-alt-PDONV but similar to PFV-alt-PDIH-PPV. Up to this point, these results reveal that introducing dialkoxyl phenylenevinylene unit into PFV based would more significantly improve the efficient emitting of LEDs than dialkoxyl naphthalenevinylene unit, and these valuable data would contribute to further design and develop new polymer LEDs.

\section{Conclusion}

Theoretical studies on the electronic structure and optical properties of PFV-alt-PDONV and PFV-alt-PDIHPPV were successfully performed. PFV-alt-PDONV shows higher twisted conformation compared to PFV-alt-PDIHPPV. Tuning the polymer backbone by adding dialkoxyl phenylenevinylene unit will more significantly stabilize LUMO and destabilized HOMO compared to dialkoxyl naphthalenevinylene unit. The calculated HOMO-LUMO gaps $\left(\Delta_{\mathrm{H}-\mathrm{L}}\right)$ and excitation energies $\left(E_{g}\right)$ are 2.77 and $2.40 \mathrm{eV}$ for PFV-alt-PDONV and 2.30 and $1.92 \mathrm{eV}$ for PFV-altPDIH-PPV, respectively. The absorption wavelengths for all oligomers are assigned to $\pi-\pi^{*}$ transition. The extrapolated absorption wavelengths of PFV-alt-PDONV and PFV-altPDIH-PPV from TDDFT method are predicted to be 516.88 and $646.09 \mathrm{~nm}$, respectively. Fluorescence energy of PFValt-PDIH-PPV exhibits red shifted compared to PFV-altPDONV. Moreover, the radiative lifetime indicates that PFValt-PDIH-PPV has longer lifetime emission than PFV-altPDONV around $0.25 \mathrm{~ns}$.

Finally, our results reveal that improving the electronic and optical properties of polymer can be controlled by the appropriate alternating group such as dialkoxyl phenylenevinylene unit which is an interesting molecule for enhancing the performance of LEDs based on easily injection of hole and electron and also long-time emission. In addition, the good agreement tendency between theoretical calculation and experiment indicates that these methods are possible and reliable for precise predicting new compounds as LEDs materials.

\section{Acknowledgments}

The authors would like to express grateful acknowledgement to Department of Chemistry, Faculty of Science, Chiang Mai University. Financial support from the Center for Innovation in Chemistry (PERCH-CIC), Commission on Higher Education, Ministry of Education is gratefully acknowledged. This work is also financially supported by The Thailand Research Fund (TRF) senior research scholar (RTA5080005). And the Graduate School of Chiang Mai University is also acknowledged. V. Sanghiran Lee, P. Nimmanpipug, R. Deang-ngern, C. Sattayanon, T. Piansawan, J. Yana, P. Tuengeun, W. Sangprasert, and C. Ngaojampa are acknowledged for their helps.

\section{References}

[1] Y. Xiong, W. Xu, C. Li et al., "Utilizing white OLED for full color reproduction in flat panel display," Organic Electronics, vol. 9, no. 4, pp. 533-538, 2008.

[2] Z. Bao, A. J. Lovinger, and J. Brown, "New air-stable n-channel organs thin film transistors," Journal of the American Chemical Society, vol. 120, no. 1, pp. 207-208, 1998.

[3] C. J. Brabec, N. S. Sariciftci, and J. C. Hummelen, "Plastic solar cells," Advanced Funtional Materials, vol. 11, no. 1, pp. 15-26, 2001.

[4] J. H. Burroughes, D. D. C. Bradley, A. R. Brown et al., "Lightemitting diodes based on conjugated polymers," Nature, vol. 347, no. 6293, pp. 539-541, 1990.

[5] Y. Ohmori, M. Uchida, K. Muro, and K. Yoshino, "Blue electroluminescent diodes utilizing poly(alkylfluorene)," Japanese Journal of Applied Physics, vol. 30, pp. L1941-L1943, 1991.

[6] M. Redecker, D. D. C. Bradley, M. Inbasekaran, and E. P. Woo, "Nondispersive hole transport in an electroluminescent polyfluorene," Applied Physics Letters, vol. 73, no. 11, pp. 15651567, 1998.

[7] D. Neher, "Polyfluorene homopolymers: conjugated liquidcrystalline polymers for bright blue emission and polarized electroluminescence," Macromolecular Rapid Communications, vol. 22, no. 17, pp. 1365-1385, 2001.

[8] U. Scherf and E. J. W. List, "Semiconducting polyfluorenestowards reliable structure-property relationships," Advanced Materials, vol. 14, no. 7, pp. 477-487, 2002.

[9] L. Yang, J. K. Feng, Y. Liao, and A. M. Ren, "A theoretical investigation on the electronic and optical properties of $\pi$ conjugated copolymers with an efficient electron-accepting unit bithieno[3,2-b:2'3'-e]pyridine," Polymer, vol. 46, no. 23, pp. 9955-9964, 2005.

[10] W. Meeto, S. Suramitr, V. Lukes, P. Wolschann, and S. Hannongbua, "Effects of the $\mathrm{CN}$ and $\mathrm{NH} 2$ substitutions on the geometrical and optical properties of model vinylfluorenes, based on DFT calculations," Journal of Molecular Structure, vol. 939, no. 1-3, pp. 75-81, 2010.

[11] S. H. Jin, H. J. Park, J. Y. Kim et al., "Poly(fluorenevinylene) derivative by Gilch polymerization for light-emitting diode applications," Macromolecules, vol. 35, no. 20, pp. 7532-7534, 2002.

[12] Z. D. Yang, J. K. Feng, and A. M. Ren, "Spiro-linked oligofluorenes and derivatives: molecular design and theoretical study of one- and two-photon absorption properties," Chemical Physics Letters, vol. 461, no. 1-3, pp. 9-15, 2008. 
[13] G. Zeng, S. J. Chua, and W. Huang, "Influence of donor and acceptor substituents on the electronic characteristics of poly(fluorene-phenylene)," Thin Solid Films, vol. 417, no. 1-2, pp. 194-197, 2002.

[14] W. Meeto, S. Suramitr, S. Vannarat, and S. Hannongbua, "Structural and electronic properties of poly(fluorene-vinylene) copolymer and its derivatives: time-dependent density functional theory investigation," Chemical Physics, vol. 349, no. 1-3, pp. 1-8, 2008.

[15] A. Donat-Bouillud, I. Levesque, Y. Tao et al., "Light-emitting diodes from fluorene-based $\pi$-conjugated polymers," Chemistry of Materials, vol. 12, no. 7, pp. 1931-1936, 2000.

[16] X. Zhou, A. M. Ren, and J. K. Feng, "Theoretical investigation on the ground- and excited-state properties of novel octupolar oligothiophene-functionalized truxenes and dipolar analogs," Polymer, vol. 45, no. 22, pp. 7747-7757, 2004.

[17] L. Yang, J. K. Feng, Y. Liao, and A. M. Ren, "Theoretical studies on the electronic and optical properties of two blue-emitting fluorene-pyridine-based copolymers," Optical Materials, vol. 29, no. 6, pp. 642-650, 2007.

[18] W. Kohn and L. J. Sham, "Self-consistent equations including exchange and correlation effects," Physical Review, vol. 140, no. 4, pp. A1133-A1138, 1965.

[19] M. Petersilka, U. J. Gossmann, and E. K. U. Gross, "Excitation energies from time-dependent density-functional theory," Physical Review Letters, vol. 76, no. 8, pp. 1212-1215, 1996.

[20] J. B. Foresman, M. Head-Gordon, J. A. Pople, and M. J. Frisch, "Toward a systematic molecular orbital theory for excited states," The Journal of Physical Chemistry, vol. 96, no. 1, pp. 135-149, 1992.

[21] C. Lee, W. Yang, and R. G. Parr, "Development of the ColleSalvetti correlation-energy formula into a functional of the electron density," Physical Review B, vol. 37, no. 2, pp. 785$789,1988$.

[22] A. D. Becke, "Density-functional thermochemistry. III. The role of exact exchange," The Journal of Chemical Physics, vol. 98, no. 7, pp. 5648-5652, 1993.

[23] K. Sriwichitkamol, S. Suramitr, P. Poolmee, and S. Hannongbua, "Structures, absorption spectra, and electronic properties of polyfluorene and its derivatives: a theoretical study," Journal of Theoretical and Computational Chemistry, vol. 5, no. 3, pp. 595-608, 2006.

[24] P. Poolmee and S. Hannongbua, "Theoretical investigation on energy gap of fluorene-thiophene copolymer," Journal of Theoretical and Computational Chemistry, vol. 3, no. 4, pp. 481489, 2004.

[25] S. Tirapattur, M. Belletête, M. Leclerc, and G. Durocher, "Study of excited state properties of oligofluorenes by the singles configuration interaction (CIS) theoretical approach," Journal of Molecular Structure, vol. 625, no. 1-3, pp. 141-148, 2003.

[26] L. Yang, A. M. Ren, J. K. Feng, and J. F. Wang, "Theoretical investigation of optical and electronic property modulations of $\pi$-conjugated polymers based on the electron-rich 3,6-dimethoxy-fluorene unit," Journal of Organic Chemistry, vol. 70, no. 8, pp. 3009-3020, 2005.

[27] P. F. Van Hutten, V. V. Krasnikov, and G. Hadziioannou, "A model oligomer approach to light-emitting semiconducting polymers," Accounts of Chemical Research, vol. 32, no. 3, pp. 257-265, 1999.

[28] K. T. Wong, C. F. Wang, C. H. Chou, Y. O. Su, G. H. Lee, and S. M. Peng, "Synthesis and properties of novel thiophenebased conjugated homologues: 9,9-diphenylfluorene-capped oligothiophenes," Organic Letters, vol. 4, no. 25, pp. 44394442, 2002.

[29] J. Ma, S. Li, and Y. Jiang, "A time-dependent DFT study on band gaps and effective conjugation lengths of polyacetylene, polyphenylene, polypentafulvene, polycyclopentadiene, polypyrrole, polyfuran, polysilole, polyphosphole, and polythiophene," Macromolecules, vol. 35, no. 3, pp. 1109-1115, 2001.

[30] G. W. T. M. J. Frisch, H. B. Schlegel, G. E. Scuseria et al., "Gaussian 03", Gaussian, Inc., Pittsburgh, PA, 2003.

[31] P. Anuragudom, Persononal Communication.

[32] S. H. Jin, S. Y. Kang, M. Y. Kim et al., "Synthesis and electroluminescence properties of poly(9,9-di-n-octylfluorenyl-2,7vinylene) derivatives for light-emitting display," Macromolecules, vol. 36, no. 11, pp. 3841-3847, 2003.

[33] S. Jungsuttiwong, S. Namuangrak, V. Promarak, T. Sudyoadsuk, and S. Vannarat, "Computer-aided design of OLED materials: a molecular modeling approach for optical properties of $\alpha$-fluorenyl oligothiophenes," Journal of Theoretical and Computational Chemistry, vol. 9, no. 6, pp. 993-1007, 2010.

[34] D. J. Tozer and N. C. Handy, "Improving virtual Kohn-Sham orbitals and eigenvalues: application to excitation energies and static polarizabilities," Journal of Chemical Physics, vol. 109, no. 23, pp. 10180-10189, 1998.

[35] C. P. Hsu, S. Hirata, and M. Head-Gordon, "Excitation energies from time-dependent density functional theory for linear polyene oligomers: butadiene to decapentaene," The Journal of Physical Chemistry A, vol. 105, no. 2, pp. 451-458, 2000.

[36] Z. L. Cai, K. Sendt, and J. R. Reimers, "Failure of densityfunctional theory and time-dependent density-functional theory for large extended $\pi$ systems," Journal of Chemical Physics, vol. 117, no. 12, pp. 5543-5549, 2002.

[37] Y. Kurashige, T. Nakajima, S. Kurashige, K. Hirao, and Y. Nishikitani, "Theoretical investigation of the excited states of coumarin dyes for dye-sensitized solar cells," The Journal of Physical Chemistry A, vol. 111, no. 25, pp. 5544-5548, 2007.

[38] B. H. Bransden and C. J. Joachain, Physics of Atoms and Molecules, Longman, London, UK, 1983. 


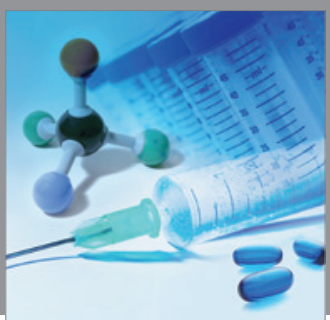

International Journal of

Medicinal Chemistry

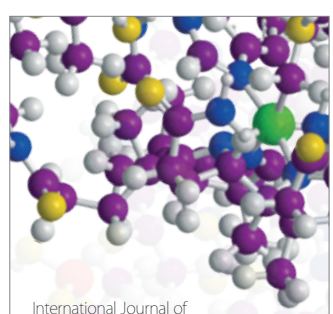

Carbohydrate Chemistry

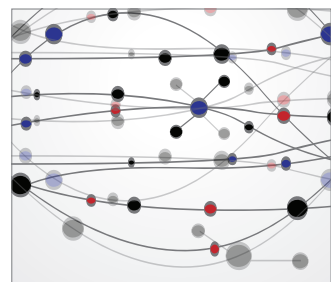

The Scientific World Journal
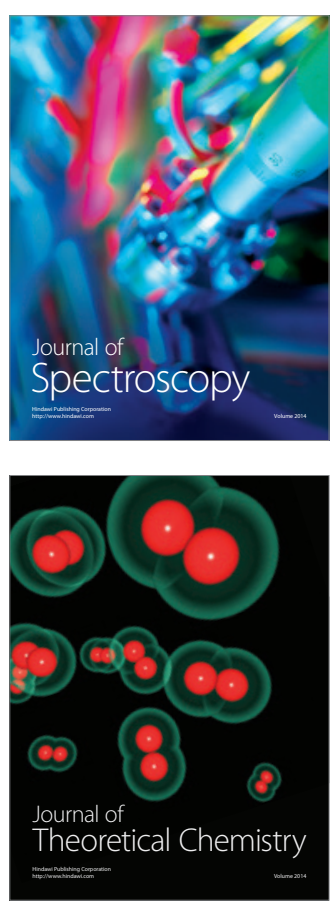
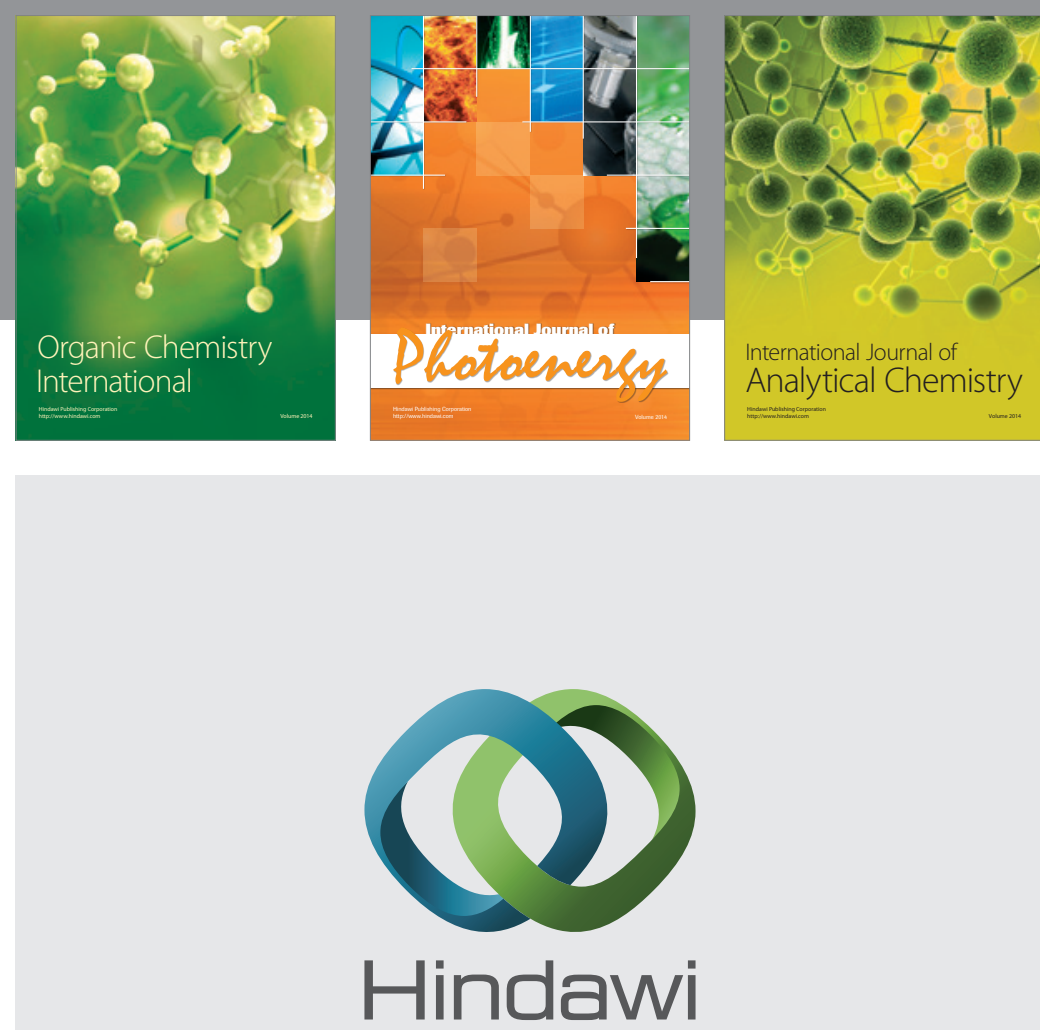

Submit your manuscripts at

http://www.hindawi.com
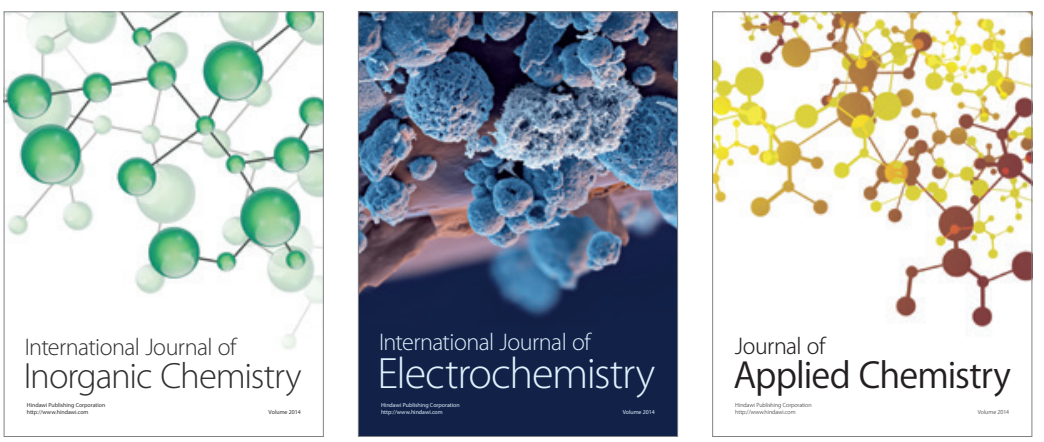

Journal of

Applied Chemistry
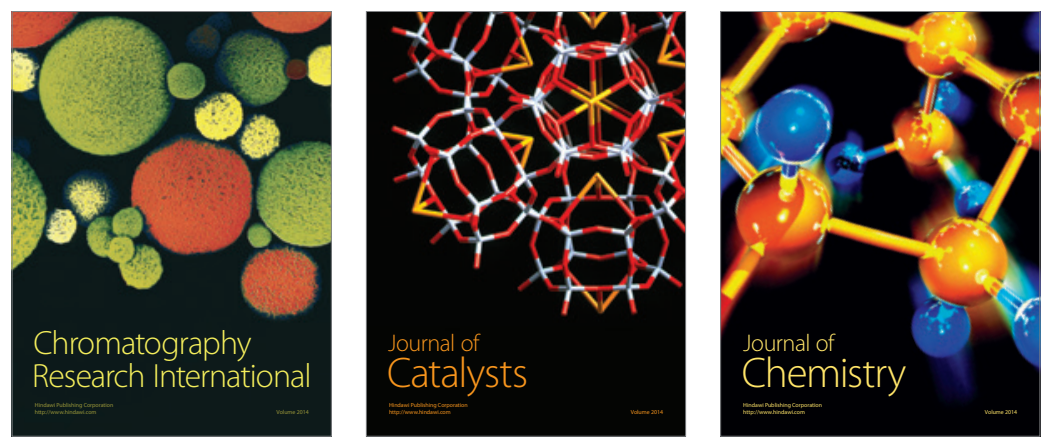
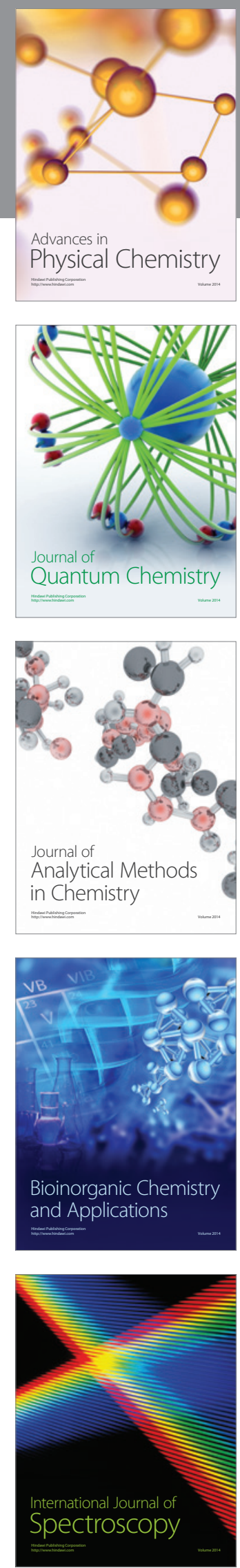